\title{
IMPACT DES JACHERES SUR LA FLORE ADVENTICE AU NORD DE LA CÔTE D'IVOIRE
}

\author{
S. SORO' ${ }^{1}, K$. A. N'GUESSAN ${ }^{2}$, D. TRAORE ${ }^{1}$ et N. OUATTARA ${ }^{3}$
}

\author{
${ }^{1}$ Université de Cocody-Abidjan, UFR Biosciences Laboratoire de Botanique 22 BP 582 Abidjan 22 Côte d'Ivoire \\ E-mail : sibirina@yahoo.fr \\ ${ }^{2}$ Central National de Recherche Agronomique, Côte d'Ivoire 10 BP 1665 Abidjan 10 Côte d'Ivoire \\ ${ }^{3}$ Ministère des Eaux et Forêts de Côte d'Ivoire 20 BP 650 Abidjan 20 Côte d'Ivoire
}

\begin{abstract}
RESUME
L'enherbement est l'un des facteurs limitants des rendements agricoles au Nord de la Côte d'Ivoire. Dans le cadre de la recherche de solutions, la flore spontanée des jachères artificielles à Légumineuses arborescentes testées est comparée à celle des jachères naturelles. Les familles les plus représentées dans la flore adventice sont les Poaceae (30\% des espèces) et les Asteraceae (14\%). La régénération d'espèces arborescentes est plus abondante dans les jachères artificielles. Inversement, les espèces adventices sont numériquement plus importantes dans les jachères naturelles (76 \% de la flore) que dans les jachères artificielles (59\%). Les jachères artificielles ont réduit l'abondance des mauvaises herbes d'environ $23 \%$.
\end{abstract}

Mots - clés : Flore adventice, rendements culturaux, jachères, légumineuses arborescentes, Côte d'Ivoire.

\section{ABSTRACT}

\section{IMPACT OF FALLOWS ON WEEDS IN NORTHERN CÔTE D'IVOIRE}

Weeds are one of the agricultural yield limiting factors in Northern Côte d'lvoire. Weed flora artificial fallows with leguminous plants tested and natural fallows were compared. Results show that represented weed families are the Poaceae (30\% of the cash) and the Asteraceae (14\%). Regeneration of tree species was more abundant in artificial fallows compared to natural fallows. Inversely, the weeds were more important in natural fallows (76 \%) compared to artificial fallows (59\%). Artificial fallows reduced weeds by $23 \%$.

Key-words : Weeds flora, yield, fallows, arborescents leguminous, Côte d'lvoire.

\section{INTRODUCTION}

L'enherbement est un problème important rencontré dans le monde agricole en général et au Nord de la Côte d'Ivoire en particulier. C'est une des causes de la baisse des rendements sur la majorité des terres cultivées. La région Nord de la Côte d'Ivoire est confrontée à des problèmes de plus en plus importants de fertilité des sols, de gestion de l'espace agro-pastoral, d'érosion, de sécurisation foncière et de production de bois de feu (Louppe, 1991 ;
Bernard, 1993). Cette dégradation de l'espace agricole est accélérée dans beaucoup de cas par l'enherbement des terres cultivées qui contribue à une baisse notable des rendements culturaux (Le Roy, 1991). Parmi les solutions envisagées à court et moyen termes, les jachères artificielles à base de Légumineuses arborescentes ont été installées et testées (Ouattara et Lebahy, 1989 ; Louppe, 1990) dans le département de Korhogo.

Le département de Korhogo est situé au nord de la Côte d'Ivoire entre $8^{\circ} 32^{\prime}$ et $10^{\circ} 20^{\prime}$ de 
latitude nord et $5^{\circ} 16^{\prime}$ et $6^{\circ} 16^{\prime}$ de longitude Ouest. La température fluctue entre $24,6^{\circ} \mathrm{C}$ en saison pluvieuse et $38^{\circ} \mathrm{C}$ en saison sèche. Le régime pluviométrique unimodal est de 6 mois. La hauteur annuelle des précipitations varie de $1019 \mathrm{~mm}$ à $1394 \mathrm{~mm}$. La zone d'étude se caractérise également par un relief constitué de plateaux tabulaires latéritiques. Les sols sont assez argileux. Mais à cause des éléments grossiers, ils ont des qualités physiques médiocres et une fertilité moyenne.

L'étude réalisée vise à déterminer l'impact des jachères naturelles et artificielles à Légumineuses arborescentes sur la richesse et la composition de la flore adventice, le taux de recouvrement et la fréquence des espèces. Elle s'inscrit dans le cadre de la recherche de techniques de lutte contre l'enherbement en zones de savanes au Nord de la Côte d'Ivoire.

\section{MATERIEL ET METHODES}

\section{MATERIEL}

Le matériel biologique est la flore adventice des jachères artificielles à base de Légumineuses arborescentes et des jachères naturelles. Les caractéristiques des jachères artificielles à base de Légumineuses arborescentes étudiées sont résumées dans le tableau 1 . Le matériel technique d'observation se compose de machettes, une corde, un ruban métrique, un appareil photographique, des sacs de récolte, une étuve de séchage.

\section{METHODES}

\section{Zone d'étude}

L'étude a été réalisée en station de recherche (jachères artificielles) et en terroirs villageois dans des jachères artificielles et naturelles. Pour les jachères artificielles, plusieurs peuplements ont été retenus. II s'agit de 7 peuplements de légumineuses arborescentes plantées en station et de 5 peuplements d'Acacia auriculiformis A. Cunn. ex Benth et d'Acacia holosericeaA. Cunn. ex G. Don. (Maslin et Thomson, 1992) installés sur des terroirs villageois. Pour les jachères naturelles, les peuplements spontanés étudiés sont contigus aux peuplements artificiels de
Légumineuses arborescentes. Ils sont répartis dans 5 terroirs villageois.

\section{Traitements}

Pour les jachères artificielles, sept peuplements de légumineuses arborescentes (en station) et 5 peuplements d'Acacia auriculiformis et d'Acacia holosericea (en terroirs villageois) ont été retenus. Pour les jachères naturelles, les peuplements spontanés étudiés sont contigus aux peuplements artificiels de légumineuses arborescentes. Ils sont répartis sur 5 terroirs villageois. Les relevés floristiques ont été réalisés sur des placettes de $25 \mathrm{~m}^{2}(5 \mathrm{~m} \times 5 \mathrm{~m})$ chacune, installées le long d'un fil de repérage tendu diagonalement dans la parcelle (N'Guessan et Tuo, 1998 ; N'Guessan et Kouamé, 2001 ; N'Guessan, 2002). Ces placettes de mesures sont équidistantes de $5 \mathrm{~m}$.

\section{Mesures et observations}

Dans chaque placette, deux paramètres ont été mesurés, l'un par comptage des individus de chaque espèce et le deuxième par estimation des taux de recouvrement de la parcelle par les espèces à partir de l'échelle de la commission des Essais Biologiques revue par Marnotte (1984). Au niveau de la flore adventice, les résultats ont été obtenus à partir des données collectées dans la végétation à l'intérieur des placettes. L'identification de certaines espèces a été faite selon les clés de détermination botanique de Hutchinson et Dalziel (1954, 1972), Lebourgeois et Merlier (1995) et d'Arbonnier (2000).

\section{Analyse des données}

Plusieurs paramètres déterminés à partir des données d'inventaires ont été retenus pour l'analyse. II s'agit de la richesse floristique, la densité des peuplements, la fréquence des individus de la flore adventice et du taux de recouvrement des espèces. La similitude entre les flores spontanées des jachères a été évaluée à l'aide du coefficient de Similitude de Sorensen (1948): $\mathrm{P}=(2 \mathrm{c} / \mathrm{a}+\mathrm{b}) \times 100$, avec $\mathrm{a}=$ le nombre d'espèces d'une liste floristique $A, b=$ le nombre d'espèces d'une liste floristique $B$ et $c=l e$ nombre d'espèces communes aux deux listes $A$ et $B$. Le coefficient générique qui exprime la richesse floristique des jachères, a été obtenu 
Tableau 1 : Caractéristiques des jachères artificielles de légumineuses arborescentes.

Characteristics of leguminous tree artificial fallows.

\begin{tabular}{lcc}
\hline $\begin{array}{l}\text { Espèces de légumineuses } \\
\text { arborescentes }\end{array}$ & $\begin{array}{c}\text { Age } \\
\text { (ans) }\end{array}$ & $\begin{array}{c}\text { Ecartements à la plantation } \\
(\mathrm{m})\end{array}$ \\
\hline Acacia auriculiformis & $13-12-10-9-8$ & $3 \times 3,5$ et $2 \times 4$ \\
Acacia colei & 8 & $2 \times 4$ \\
Acacia crassicarpa & 12 & $3 \times 3,5$ \\
Acacia holosericea & 8 & $2 \times 4$ \\
Acacia neurocarpa & 8 & $2 \times 4$ \\
Albizia guachepele & 7 & $2 \times 4$ \\
Albizia zygia & 12 & $3 \times 3,5$ \\
\hline
\end{tabular}

à l'aide de rapport du nombre total de genres et des espèces qui leur appartiennent selon la formule suivante : $\mathrm{Cg}=\mathrm{G} / \mathrm{E}$, avec $\mathrm{G}=\mathrm{le}$ nombre de genres et $\mathrm{E}=$ le nombre des espèces (Aké Assi, 1984).

\section{RESULTATS}

Les résultats présentent les caractéristiques de la flore adventice dans les jachères naturelles et artificielles. Ces caractéristiques sont : richesse floristique; de recouvrement du sol et de densités des espèces, de fréquence relative des espèces, de coefficients de similitude entre les flores et de coefficients génériques des flores spontanées.

\section{FLORE ADVENTICE DANS LES JACHERES NATURELLES}

\section{Richesse floristique et densités des espèces de la flore adventice}

Les résultats de l'étude de la flore adventice sont montrés par les tableaux 2 et 3 et illustrés par la figure 1. La flore adventice est composée de 18 familles, 38 genres et de 46 espèces. Les familles les mieux représentées par ordre d'importance quantitative (regroupant au moins $80 \%$ des espèces) sont : les Poaceae, Asteraceae, Malvaceae, Fabacaceae et Euphorbiaceae. Les Poaceae sont plus riches en espèces. On rencontre en moyenne 5 à 11 espèces par placette de $25 \mathrm{~m}^{2}$. Les Poaceae sont représentées majoritairement par 7 espèces très fréquentes. Ce sont: Andropogon gayanus Kunth., Hyparrhenia involucrata Stapf., Digitaria horizontalis Willd., Imperata cylindrica L., Pennisetum polystachion L., Eragrostis ciliaris (L.) R. Brown et Paspalum scrobiculatum Berg. Les Astéracées sont représentées par 4 espèces fréquentes: Acanthospermum hispidum De Candolle, Ageratum conyzoides Benth., Aspilia bussei Oliv. et Hiren et Tridax procumbens L. Les autres familles sont représentées pour la plupart par une ou deux espèces.

\section{Recouvrement du sol et fréquence relative des espèces de la flore adventice}

Les résultats des inventaires et analyses sont consignés dans le tableau 4. Les espèces qui possèdent les taux de couverture du sol les plus élevés (> $50 \%$ ) sont Acanthospermum hispidum, Imperata cylindrica et Tricliceras pilosum (Will.) Persoon. Pour les autres espèces étudiées, ces taux de couverture du sol sont inférieurs à $30 \%$.

Parmi les espèces rencontrées régulièrement, trois espèces possèdent les fréquences relatives les plus élevées (10\%) ; il s'agit d'Andropogon gayanus, Cassia mimosoides L. et d'Hyparrhenia involucrata. On rencontre, à une fréquence de moindre ( $9 \%)$, plusieurs espèces parmi lesquelles Aspilia bussei, Paspalum scrobiculatum et Tephrosia bracteolata Guill. et Perr. 
Tableau 2 : Familles de la flore adventice dans les jachères naturelles (nombre). Weed flora families in natural fallows (number).

\begin{tabular}{lcc}
\hline Familles & Genres & Espèces \\
\hline Araceae & 1 & 1 \\
Asteraceae & 5 & 6 \\
Césalpiniaceae & 1 & 2 \\
Commelinaceae & 1 & 1 \\
Convolvulaceae & 1 & 1 \\
Cyperaceae & 1 & 1 \\
Euphorbiaceae & 2 & 3 \\
Fabaceae & 4 & 4 \\
Lamiaceae & 1 & 1 \\
Loganiaceae & 1 & 1 \\
Malvaceae & 3 & 4 \\
Mimosaceae & 1 & 1 \\
Poaceae & 11 & 13 \\
Rubiaceae & 1 & 3 \\
Scrofulariaceae & 1 & 1 \\
Sterculiaceae & 1 & 1 \\
Tiliaceae & 1 & 1 \\
Turneraceae & 1 & 1 \\
\hline
\end{tabular}

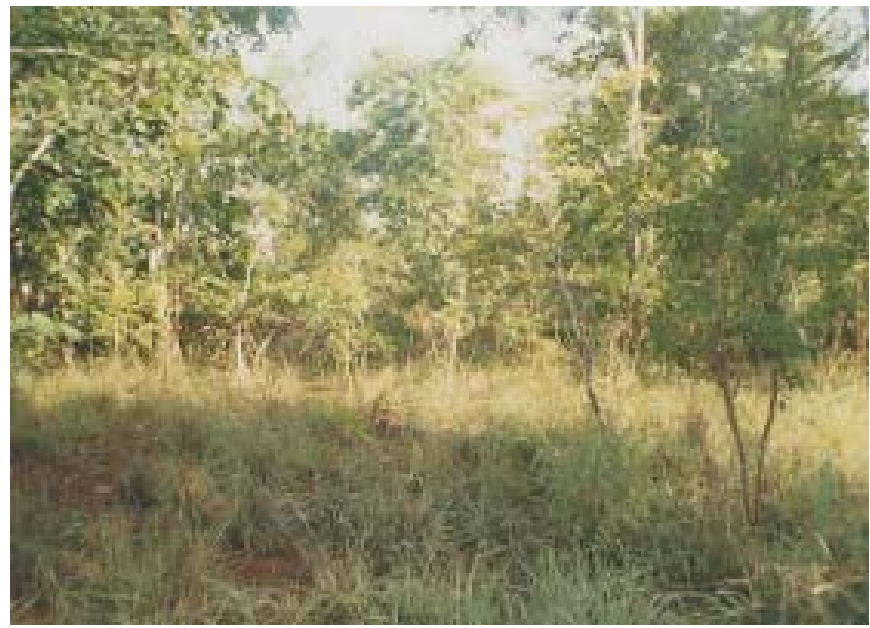

Figure 1 : Flore adventice sous jachère naturelle. Weed flora in the natural fallow. 
Tableau 3 : Espèces adventices des jachères naturelles.

Weed species of natural fallows.

\begin{tabular}{|c|c|}
\hline Familles & Espèces \\
\hline Araceae & Stylochiton hypogaeus Lepr. \\
\hline Asteraceae & Acanthospermum hispidum De Candolle \\
\hline Asteraceae & Ageratum conyzoides Benth. \\
\hline Asteraceae & Aspilia bussei Oliv. et Hiren. \\
\hline Asteraceae & Aspilia kotschyi Oliver \\
\hline Asteraceae & Tridax procumbens $\mathrm{L}$. \\
\hline Asteraceae & Vernonia ambigua Kotschy et Peyritsch \\
\hline Caesalpiniaceae & Cassia mimosoides $\mathrm{L}$. \\
\hline Caesalpiniaceae & Cassia obtusifolia $\mathrm{L}$. \\
\hline Commelinaceae & Commelina benghalensis $\mathrm{L}$. \\
\hline Convolvulaceae & Ipomoea eriocarpa $\mathrm{R} . \mathrm{Br}$. \\
\hline Cyperaceae & Cyperus rotundus $\mathrm{L}$. \\
\hline Euphorbiaceae & Euphorbia heterophylla $\mathrm{L}$. \\
\hline Euphorbiaceae & Euphorbia hirta $\mathrm{L}$. \\
\hline Euphorbiaceae & Phyllanthus amarus Sch. et Th. \\
\hline Fabaceae & Desmodium dichotomum \\
\hline Fabaceae & Stylosanthes hamata \\
\hline Fabaceae & Tephrosia bracteolata Guill. et Perr. \\
\hline Fabaceae & Zornia glochidiata Reichb. ex DC \\
\hline Lamiaceae & Hyptis suaveolens Poit. \\
\hline Loganiaceae & Spigelia anthelmia \\
\hline Malvaceae & Hibiscus asper Hook. f. \\
\hline Malvaceae & Sida acuta Brum.F \\
\hline Malvaceae & Sida urens Brum.F \\
\hline Malvaceae & Urena lobata \\
\hline Mimosaceae & Mimosa pudica \\
\hline Poaceae & Andropogon gayanus Kunth. \\
\hline Poaceae & Brachiaria vilosa (Lamarck) A.Camus \\
\hline Poaceae & Dactyloctenium aegyptium \\
\hline Poaceae & Digitaria horizontalis Willd. \\
\hline Poaceae & Eragrostis aspera (Jacquin) Nees \\
\hline Poaceae & Eragrostis ciliaris (L.) R.Brown \\
\hline Poaceae & Hyparrhenia involucrata Stapf. \\
\hline Poaceae & Imperata cylindrica $\mathrm{L}$. \\
\hline Poaceae & Mnesithea granularis L. \\
\hline Poaceae & Paspalum scrobiculatum Berg \\
\hline Poaceae & Pennisetum pedicellatum Trin. \\
\hline Poaceae & Pennisetum polystachion $\mathrm{L}$. \\
\hline Poaceae & Sporobolus indicus L. \\
\hline Rubiaceae & Spermacoce chaetocephala De Candolle \\
\hline Rubiaceae & Spermacoce radiata De Candolle \\
\hline Rubiaceae & Spermacoce stachytea De Candolle \\
\hline Scrofulariaceae & Striga hermontheca (Del.) Benth. \\
\hline Sterculiaceae & Waltheria indica $\mathrm{L}$. \\
\hline Tiliaceae & Corchorus olitorius L. \\
\hline Turneraceae & Tricliceras pilosum (Will.) Persoon \\
\hline
\end{tabular}


Tableau 4 : Taux de recouvrement (\%) et fréquence relative des espèces de la flore adventice dans les jachères naturelles.

Recovery rates (\%) and relative frequency of weed flora in natural fallows.

\begin{tabular}{|c|c|c|}
\hline Espèces & $\begin{array}{c}\text { Taux de recouvrement } \\
(\%)\end{array}$ & Fréquence relative \\
\hline Acanthospermum hispidum & 70 & 6 \\
\hline Ageratum conyzoides & & 6 \\
\hline Andropogon gayanus & 15 & 10 \\
\hline Aspilia bussei & & 9 \\
\hline Cassia mimosoides & 15 & 10 \\
\hline Corchorus olitorius & 15 & 6 \\
\hline Commelina benghalensis & 15 & \\
\hline Cyperus rotundus & 15 & 6 \\
\hline Digitaria horizontalis & 15 & 6 \\
\hline Eragrostis ciliaris & & 9 \\
\hline Euphorbia hirta & & 6 \\
\hline Hyparrhenia involucrata & 30 & 10 \\
\hline Hyptis suaveolens & 30 & 8 \\
\hline Imperata cylindrica & 85 & \\
\hline Ipomoea eriocarpa & & 6 \\
\hline Paspalum scrobiculatum & 15 & 9 \\
\hline Pennisetum polystachion & & 9 \\
\hline Sida acuta & & 9 \\
\hline Sida urens & 15 & 9 \\
\hline Spermacoce chaetocephala & & 9 \\
\hline Spermacoce radiata & 30 & 8 \\
\hline Tephrosia bracteolata & 30 & 9 \\
\hline Tricliceras pilosum & 70 & \\
\hline Tridax procumbens & 15 & \\
\hline Waltheria indica & & 9 \\
\hline Zornia glochidiata & & 9 \\
\hline
\end{tabular}

\section{FLORE ADVENTICE DANS LES JACHERES ARTIFICIELLES}

\section{Richesse floristique et densités des espèces de la flore adventice}

Les données d'inventaires floristiques sont résumées dans les tableaux 5 et 6 et illustrés par la figure 2. La flore adventice identifiée sous les peuplements de légumineuses arborescentes comprend 17 familles, 37 genres et 44 espèces.

Les jachères artificielles réduisent la densité (abondance) des mauvaises herbes d'environ $23 \%$. La distribution de ces végétaux est hétérogène et varie d'un site à l'autre. Les familles les mieux représentées en espèces sont les Poaceae (30\%) et les Asteraceae (16\%). A un degré de moindre d'importance (7 à $14 \%$ ), on rencontre les Euphorbiaceae, Fabaceae, Rubiaceae et les Malvaceae.

Les familles des Commelinaceae, Solanaceae, Taccaceae et des Verbenaceae sont les plus faiblement représentées en espèce ( 2 à 5 p.c. d'espèces par famille). Sur le plan de la Densité de la flore, on obtient 3 à 9 espèces d'adventices par placette de 25 $\mathrm{m}^{2}$. Les espèces de la famille des Poaceae les plus couramment rencontrées dans les jachères artificielles à légumineuses arborescentes sont : Andropogon gayanus, Imperata cylindrica, Rottboellia cochinchinensis Loureito et Pennisetum polystachion. 
Tableau 5 : Familles de la flore adventice des jachères artificielles (nombre).

Weed flora families in artificial fallows (number).

\begin{tabular}{lcc}
\hline Familles & Genres & Espèces \\
\hline Amaranthaceae & 1 & 1 \\
Asteraceae & 6 & 7 \\
Caesalpiniaceae & 1 & 2 \\
Commelinaceae & 1 & 2 \\
Convolvulaceae & 1 & 2 \\
Cyperaceae & 2 & 2 \\
Euphorbiaceae & 2 & 4 \\
Fabaceae & 5 & 6 \\
Lamiaceae & 2 & 2 \\
Loganiaceae & 1 & 1 \\
Malvaceae & 3 & 4 \\
Mimosaceae & 1 & 1 \\
Poaceae & 11 & 12 \\
Rubiaceae & 2 & 3 \\
Smilacaceae & 1 & 1 \\
Solanaceae & 1 & 1 \\
Sterculiaceae & 1 & 1 \\
Tiliaceae & 1 & 1 \\
Taccaceae & 2 & 2 \\
Verbenaceae & 1 & 1 \\
\hline
\end{tabular}

Tableau 6 : Espèces adventices des jachères artificielles à légumineuses arborescentes.

Weed species of leguminous trees in artificial fallows.

\begin{tabular}{ll}
\hline Familles & Espèces \\
\hline Asteraceae & Ageratum conyzoides \\
Asteraceae & Tridax procumbens \\
Commelinaceae & Commelina benghalensis \\
Convolvulaceae & Ipomoea eriocarpa \\
Euphorbiaceae & Euphorbia hirta \\
Euphorbiaceae & Phyllanthus amarus \\
Fabacaceae & Tephrosia bracteolata \\
Fabaceae & Zornia glochidiata \\
Lamiaceae & Hyptis suaveolens \\
Lamiaceae & Leucas martinicensis (Jacquin) R. Brown \\
Loganiaceae & Spigelia anthelmia \\
Malvaceae & Sida urens \\
Poaceae & Andropogon gayanus \\
Poaceae & Digitaria horizontalis \\
Poaceae & Eragrostis ciliaris \\
Poaceae & Imperata cylindrica \\
Poaceae & Paspalum scrobiculatum \\
Poaceae & Pennisetum pedicellatum \\
Poaceae & Pennisetum polystachion \\
Poaceae & Rottboellia cochinchinensis \\
Rubiaceae & Spermacoce chaetocephala \\
Rubiaceae & Spermacoce radiata \\
Turneraceae & Tricliceras pilosum \\
Sterculiaceae & Waltheria indica \\
\hline
\end{tabular}




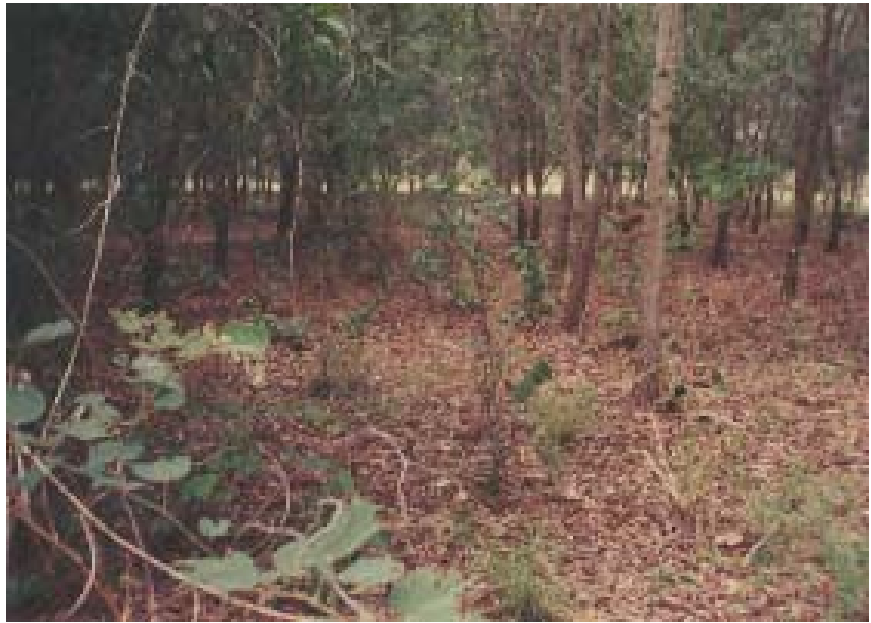

Figure 2 : Flore adventice sous jachère artificielle à Acacia australien.

Weed flora in artificial fallow of australian acacia.

\section{Recouvrement et fréquence relative des espèces de la flore adventice}

Les résultats des inventaires et analyses des données sont résumés dans les tableaux 7 et 8. Andropogon gayanus est présente dans toutes les jachères de légumineuses arborescentes étudiées, avec des taux de recouvrement variant entre $30 \%$ (dans les jachères à Acacia auriculiformis et Acacia neurocarpa A. Cunn. ex Hook (Maslin et Thomson, 1992)) et $15 \%$ dans les autres jachères. Imperata cylindrica, Pennisetum polystachion et Rottboellia cochinchinensis sous Acacia colei Maslin et L. A. J. Thomson (Maslin et Thomson, 1992), Acacia holosericea et sous Albizia guachepele Kunt Dug. avec des taux de couverture compris entre 30 et $50 \%$ sont rencontrés.

Tridax procumbens a été uniquement observé sous les peuplements d'Acacia auriculiformis et Acacia colei avec des taux de couverture respectifs de 30 et $15 \%$.

Les taux de présence et de couverture diffèrent en fonction des espèces. Au niveau de la fréquence, Andropogon gayanus est plus régulière sous Acacia crassicarpa A.Cunn. ex Benth (16\%) que sous les autres légumineuses arborescentes. Aspilia bussei a été plus fréquent sous Acacia auriculiformis et l'Acacia crassicarpa avec un taux de couverture de $16 \%$. Quant à Rottboellia cochinchinensis, elle a été retrouvée beaucoup plus sous Acacia crassicarpa qu'ailleurs. Imperata cylindrica a été faiblement présente sous Acacia auriculiformis.

\section{COMPARAISON DES CARACTERISTIQUES FLORISTIQUES DES JACHERES}

\section{Richesse floristique}

Les analyses comparatives des données d'inventaires dans les différents types de jachères ont donné les résultats consignés dans le tableau 9 . Les jachères artificielles ont eu une flore spontanée plus diversifiée, un tapis herbacé clairsemé avec des plantes très dispersées qu'en jachères naturelles (Figures $1 ; 2$ ). La régénération d'espèces arborescentes a été plus abondante dans les jachères artificielles (42\%) que dans les jachères naturelles (24\%). Inversement, la flore adventice a été plus importante dans les jachères naturelles $(76 \%)$ que dans les jachères artificielles (59\%).

La décroissance de la diversité de la flore spontanée des jachères artificielles en station, en milieu paysan aux jachères naturelles traduit la qualité de la gestion dont elles ont été sujettes en fonction de leur localisation. D'une protection intégrale des jachères artificielles contre les feux de brousse, le pâturage et les prélèvements anarchiques des organes végétaux en station, on est passé à une gestion peu contrôlée voire libre des jachères artificielles paysannes et naturelles. 


\section{Similitude entre les flores spontanées des jachères}

Les coefficients de similitude de la flore spontanée dans les différents types de jachères étudiés sont résumés dans le tableau 10. Le coefficient de similitude est égal à $57 \%$, entre les jachères naturelles et les jachères artificielles (station) ; à $62 \%$ entre les jachères naturelles et les jachères artificielles (paysannes). Un coefficient de similitude de $58 \%$ entre les jachères artificielles (paysannes) et les jachères artificielles (station) a été obtenu.

Les flores spontanées ont diffèré les unes des autres en fonction des types de jachères, de leur localisation et de la qualité de leur gestion (réglementée ou libre).

\section{Coefficients génériques des flores spontanées}

Les résultats des analyses des données sont consignés dans le tableau 11. En jachères artificielles, les coefficients génériques sont de 0,838 (soit $84 \%$ ) pour les jachères de station de recherche et 0,876 (soit $88 \%$ ) pour les jachères paysannes. En jachères naturelles, le coefficient générique est de 0,866 (soit $87 \%$ ). Dans les trois types de jachères, environ une espèce par genre a été obtenue.

Tableau 7 : Taux de recouvrement des espèces de la flore adventice dans les jachères artificielles (\%). Recovery rates of weed species in artificial fallows (\%).

\begin{tabular}{|c|c|c|c|c|c|c|}
\hline \multirow[b]{2}{*}{ Espèces } & \multicolumn{6}{|c|}{ Leguminous tree species } \\
\hline & $\begin{array}{c}\text { Acacia } \\
\text { auriculiformis }\end{array}$ & $\begin{array}{c}\text { Acacia } \\
\text { colei }\end{array}$ & $\begin{array}{c}\text { Acacia } \\
\text { crassicarpa }\end{array}$ & $\begin{array}{c}\text { Acacia } \\
\text { holosericea }\end{array}$ & $\begin{array}{c}\text { Albizia } \\
\text { guachepele }\end{array}$ & $\begin{array}{c}\text { Acacia } \\
\text { neurocarpa }\end{array}$ \\
\hline Andropogon gayanus & 30 & 15 & & 15 & 15 & 30 \\
\hline Aspilia bussei & 15 & & & & & \\
\hline Aspilia kotschyi & 15 & & & & & \\
\hline Digitaria horizontalis & 15 & & & & & \\
\hline Euphorbia heterophylla & 15 & & & & & \\
\hline Hyptis suaveolens & 15 & & & 15 & & \\
\hline Imperata cylinrica & 15 & 30 & & 30 & & 50 \\
\hline Ipomoea eriocarpa & 15 & & & & & \\
\hline Pennisetum polystachion & 50 & 50 & & 50 & & 30 \\
\hline Rottboellia cochinchinensis & 15 & 30 & 50 & & 50 & \\
\hline Sida urens & 15 & & & & & \\
\hline Spermacoce chaetocephala & 15 & & & 30 & & \\
\hline Tridax procumbens & 30 & 15 & & & & \\
\hline
\end{tabular}

Tableau 8 : Fréquence relative (\%) des espèces de la flore adventice dans les jachères artificielles. Relative frequency (\%) of weed species in artificial fallows.

\begin{tabular}{|c|c|c|c|c|c|c|}
\hline \multirow[b]{2}{*}{ Espèces } & \multicolumn{6}{|c|}{ Leguminous tree species } \\
\hline & $\begin{array}{c}\text { Acacia } \\
\text { auriculiformis }\end{array}$ & $\begin{array}{c}\text { Acacia } \\
\text { colei }\end{array}$ & $\begin{array}{c}\text { Acacia } \\
\text { crassicarpa }\end{array}$ & $\begin{array}{c}\text { Acacia } \\
\text { holosericea }\end{array}$ & $\begin{array}{c}\text { Albizia } \\
\text { guachepele }\end{array}$ & $\begin{array}{c}\text { Acacia } \\
\text { neurocarpa }\end{array}$ \\
\hline Andropogon gayanus & 12 & & 16 & 12 & & \\
\hline Aspilia bussei & & & 16 & & 13 & \\
\hline Aspilia kotschyi & 16 & & & & & \\
\hline Imperata cylinrica & 14 & & & & & 13 \\
\hline Pennisetum polystachion & 12 & 12 & & 12 & 13 & 13 \\
\hline Rottboellia cochinchinensis & 15 & 12 & 16 & 9 & 13 & \\
\hline Sida urens & & & & & 13 & \\
\hline Spermacoce chaetocephala & & & & 9 & & \\
\hline
\end{tabular}


Tableau 9 : Caractéristiques floristiques globales des jachères.

Global floral characteristics of fallows.

\begin{tabular}{lccc}
\hline & \multicolumn{3}{c}{ Types de jachères } \\
\cline { 2 - 4 } Paramètres & $\begin{array}{c}\text { Jachères artificielles } \\
\text { (station) }\end{array}$ & $\begin{array}{c}\text { Jachères artificielles } \\
\text { (paysanne) }\end{array}$ & Jachères naturelles \\
\hline Nombre familles & 40 & 35 & 28 \\
Flore globale (nombre espèces) & 90 & 81 & 62 \\
Espèces arborescentes (\%) & 51 & 32 & 24 \\
Espèces adventices $(\%)$ & 49 & 68 & 76 \\
\hline
\end{tabular}

Tableau 10 : Coefficients de similitude entre les jachères.

Similarity coefficients between fallows.

\begin{tabular}{|c|c|}
\hline Jachères & $\begin{array}{l}\text { Coefficients de similitude } \\
(\%)\end{array}$ \\
\hline $\begin{array}{l}\text { Jachères naturelles } \\
\text { paysannes }\end{array}$ & 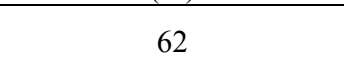 \\
\hline $\begin{array}{l}\text { jachères artificielles paysannes et jachères } \\
\text { artificielles en station de recherche }\end{array}$ & 58 \\
\hline $\begin{array}{l}\text { Jachères naturelles et jachères artificielles en } \\
\text { station de recherche }\end{array}$ & 57 \\
\hline
\end{tabular}

Tableau 11 : Coefficients génériques de la flore adventice dans les jachères.

Generic coefficients of weed flora in fallows.

\begin{tabular}{lccc}
\hline Jachères & Coefficients génériques & Valeurs en \% & Nombre d'espèces / genre \\
\hline \multirow{2}{*}{ Jachères artificielles } & 0,838 (station) & 84 (station) & 1,19 \\
& 0,876 (paysannes) & 88 (paysannes) & 1,14 \\
Jachères naturelles & 0,866 & 87 & 1,15 \\
\hline
\end{tabular}

\section{DISCUSSION}

Les coefficients génériques de la flore spontanée obtenus ont été identiques à ceux des études réalisées aux alentours de Korhogo au Nord de la Côte d'Ivoire par Koulibaly (2000) avec une valeur moyenne de $83 \%$. La comparaison des coefficients génériques de cette flore spontanée avec celui des études effectuées à Lamto au centre de la Côte d'Ivoire (Kouamé, 1993) montre que la flore spontanée des jachères du Nord est pauvre. Ces études ont donné $42 \%$ de coefficient générique contre $86 \%$ en moyenne pour les jachères étudiées. Ce constat peut être imputable à la nature et à la composition floristique de la savane moins riche en espèces que la zone de Lamto située en zone préforestière.

La richesse floristique globale des jachères artificielles est plus élevée que celle des jachères naturelles. Les espèces de la flore adventice les plus abondantes et représentatives sont Andropogon gayanus, Hyparrhenia involucrata, Imperata cylindrica, Rottboellia cochinchinensis et Ageratum conyzoides. Ces résultats obtenus sous les jachères artificielles à légumineuses arborescentes et dans les jachères naturelles, de même âge, ont été confirmés par les études menées par Koulibaly (2000) aux alentours de la ville de Korhogo sur la flore de la savane soudanienne. Ces deux études ont aussi montré que les familles des Poaceae et des Asteraceae sont les plus importantes de la flore adventice du département de Korhogo.

La valeur de la similitude entre les flores spontanées de 2 jachères a varié avec les caractéristiques de ces dernières : type biologique des espèces ligneuses en peuplement ; taux de couverture du sous bois et âge du peuplement. La composition de la flore spontanée, d'une jachère à l'autre, a été 
également influencée par le facteur anthropique à travers les activités des populations en leur sein : protection; mise en culture partielle ; pâturage ; exploitation de bois et feux de brousse. Certaines jachères artificielles paysannes et les jachères artificielles en station de recherche sont proches parce qu'elles bénéficient de protection. Aussi, en milieu paysan, certaines jachères artificielles et les jachères naturelles sont-elles également proches dans leur composition floristique parce que plus ou moins exploitées.

La jachère artificielle à base de Légumineuse arborescente, bien entretenue, a un effet dépressif et sélectif sur le développement de la flore adventice. L'ombrage excessif du peuplement de la Légumineuse arborescente empêche plus ou moins le développement des espèces adventices héliophiles envahissant les espaces découverts. Parallèlement, la jachère artificielle à base de Légumineuse arborescente bien protégée (station de recherche) augmente doublement la biodiversité des espèces ligneuses spontanées. Sur le sol fertile constitué de la matière organique épaisse (litière de la Légumineuse arborescente) plus ou moins décomposée et à l'ombre des arbres, les graines des espèces ligneuses germent facilement dans la jachère artificielle à base de Légumineuses arborescentes. Les semences des espèces ligneuses y sont aussi dispersées par des agents disséminateurs animaux qui fréquentent le sous bois et le feuillage ombragés du peuplement de la Légumineuse arborescente (oiseaux, roussettes et autres animaux).

\section{CONCLUSION}

Si elle est bien conduite, la pratique de la jachère artificielle à base de légumineuses arborescentes contribue à l'accroissement de la biodiversité des espèces ligneuses spontanées et à une réduction de la flore adventice. L'enherbement est responsable de profondes diminutions des rendements culturaux dans le monde en général et au Nord de la Côte d'Ivoire en particulier. La baisse de l'enherbement est d'autant plus importante que les plantations de légumineuses arborescentes sont mieux entretenues (N'Guessan, 2002).

Tout en réduisant le potentiel de régénération de la flore adventice, ces Légumineuses arborescentes restaurent la fertilité des sols par le biais de la fixation de l'azote atmosphérique
(Bonnemain et Dumas, 1998 ; Dommergues et al., 1999) et de la litière (N'Guessan, 1991). Elles sont également sources de bois d'énergie dont la pénurie se présente avec acuité au Nord de la Côte d'Ivoire.

Les jachères naturelles et artificielles sont deux méthodes importantes dans la restauration des terres agraires. Mais aujourd'hui, la pression foncière engendrée par la pression humaine dans cette région du pays ne permet plus la pratique de la jachère naturelle de longue durée nécessaire à une meilleure reconstitution du sol de culture. Les jachères artificielles à base de Légumineuses arborescentes se présentent comme de bonnes alternatives en raison de leur grande potentialité fertilisante à court ou moyen terme si le choix des espèces est bien fait.

Cette étude représente aussi une contribution à une meilleure connaissance de la flore et de la biodiversité du Nord de la Côte d'Ivoire.

\section{REFERENCES}

Aké Assi (L.). 1984. Flore de la Côte d'Ivoire. Etude descriptive et biogéographique avec quelques notes ethnobotaniques. Thèse de Doctorat d'Etat ès Sciences Naturelles, Faculté des Sciences et Techniques, Univ. Nationale de Côte d'Ivoire, 3 tomes, 6 volumes, $1205 \mathrm{p}$.

Arbonnier (M.). 2000. Arbres, arbustes et lianes dans les zones sèches d'Afrique de l'Ouest. Edition CIRAD, CIRAD/ MNHN/ UICN, $541 \mathrm{p}$.

Bernard (C.). 1993. Typologie d'un village sénoufo en zone dense. Cas de Dolékaha dans la région de Korhogo (Côte d'Ivoire). DESS Gestion des systèmes agro-sylvopastoraux en zone tropicale, Univ. Paris Val de Marne, 100 p. + annexes + cartes.

Bonnemain (J. L.) et (C.) Dumas. 1998. La biologie végétale. Presses Universitaires France. Paris, $125 \mathrm{p}$.

Coulibaly (S.). 1978. Le paysan sénoufo. Nouvelles Editions Africaines (NEA), Abidjan, 245 p.

Daubrey (A.) et (P. R.) Akassey. 1992. La terre et l'histoire. Guide de l'agriculture en Côte d'Ivoire, Edition PRAT/EUROPA, Volume I, pp. 21 - 55.

Dommergues (Y.) ; Duhoux (E.) et (G.) Hoang. 1999. Les arbres fixateurs. Caractéristiques fondamentales et rôles dans l'aménagement des écosystèmes méditerranéens et tropicaux. Edition CIRAD/FAO/ IRD, 499 p. 
Hutchinson (J.) et (J. M.) Dalziel. 1954 et 1972. Flora of West Tropical Africa, Ed. $2^{\mathrm{e}}$ by KEAY, $R$ and HEPPER F.N., Crown Agents for Oversea Governments and Administrations, Londi, 3 volumes.

Kouamé (N. F.). 1993. Contribution au recensement des Monocotylédones de la réserve de Lamto (Côte d'Ivoire) et à la connaissance de leur place dans les différents faciès savaniens. Mém. DEA, Univ. de Côte d'Ivoire, $128 \mathrm{p}$.

Koulibaly (A. V.). 2000. Caractéristiques de la végétation et de la flore de la savane soudanienne de Côte d'lvoire : cas des alentours de la ville de Korhogo. Mém. DEA, Univ. de Cocody, Côte d'Ivoire, 70 p. + annexes.

Le Bourgeois (T.) et (H.) Merlier. 1995. Les adventices d'Afrique soudano-sahelienne. Adventrop. Edition CIRAD - CA, 640 p.

Le Roy (X.). 1991. Pratique de la jachère dans les terroirs Sénoufo du nord de la Cote d'Ivoire. Actes de l'Atelier International sur la jachère en Afrique de l'Ouest, Montpellier, 3-5 décembre 1991, $15 \mathrm{p}$.

Louppe (D.). 1990. Sylviculture intensive en zones de savanes. Séminaire UNESCO de Korhogo sur la Productivité des savanes, mai 1990, Côte d'Ivoire, 10 p. + 6 fig.

Louppe (D.). 1991. Recherche scientifique et développement des productions forestières en zone de savanes. $4^{\mathrm{e}}$ Assises biennales de l'Association Ivoirienne des Sciences Agronomiques (AISA), Korhogo, 20-25 novembre 1991, $13 \mathrm{p}$.
Marnotte (P.). 1984. Echelle de la commission des Essais Biologiques (CEB). Echelle revue en 1984. Fiches techniques, CIRAD, $4 \mathrm{p}$.

Maslin (B. R.) et (L. A. J.) Thomson. 1992. Reappraisal of the taxonomy of Acacia holosericea A.Cun. ex Don, including the description of a new species, $A$. colei and the reinstatement of $A$. neurocarpa $\mathrm{A}$. Cun. Ex Hook. Australian Systematic Botany, 5:729-743.

N'Guessan (K. A.). 1991. Contribution à l'étude de méthodes de régénération des jachères en basse Côte d'Ivoire : le cas de l'Acacia mangium. Thèse de Docteur-Ingénieur. Université Nationale de Côte d'Ivoire. Faculté des Sciences et Techniques, $111 \mathrm{p}$.

N'Guessan (A.) et (Y.) Kouamé. 2001. Bilan des activités de recherches agroforestières au Nord de la Côte d'Ivoire. Rap. scien., Direction Régionale du CNRA de Korhogo, $9 \mathrm{p}$.

N'Guessan (A.) et (N.) Tuo. 1998. La flore sous les cocoteraies en basse Côte d'lvoire. Rap. scien., CNRA de Côte d'Ivoire, 7 p.

Ouattara (N.) et (C.) Lebahy. 1989. Plantations expérimentales à Acacia auriculaeformis en 1988. Analyses de premières mensurations effectuées à 6 mois. Rap. scien., CTFT de Côte d'Ivoire, 8 p.

Sorensen (T.). 1948. A method of establishing group of equal amplitude in plant sociology based on similary of species content. Det Kongelige Danske Videns Kabernes Selskab, Biologiske Skrifter, 5, 4 ; 1-34 p. 
ANNEXE

\section{Liste alphabétique des taxons cités}

Acacia auriculiformis A. Cunn. ex Benth. (Mimosaceae)

Acacia colei Maslin et L.A.J. Thomson (Mimosaceae)

Acacia crassicarpa A. Cunn. ex Benth (Mimosaceae)

Acacia holosericea A. Cunn. ex G. Don (Mimosaceae)

Acacia neurocarpa A. Cunn. ex Hook (Mimosaceae)

Acanthospermum hispidum De Candolle (Asteraceae)

Ageratum conyzoides Benth. (Asteraceae)

Albizia guachepele Kunth Dug (Mimosaceae)

Albizia zygia DC J.F. Macabor (Mimosaceae)

Andropogon gayanus Kunth. (Poaceae)

Aspilia bussei Oliv. et Hiren. (Asteraceae)

Cassia mimosoides L. (Caesalpiniaceae)

Commelina benghalensis L. (Commelinaceae)

Corchorus olitorius L. (Tiliaceae)

Cyperus rotundus L. (Cyperaceae)

Digitaria horizontalis Willd. (Poaceae)

Eragrostis ciliaris (L.) R. Brown (Poaceae)
Euphorbia hirta L. (Euphorbiaceae)

Hyparrhenia involucrata Stapf (Poaceae)

Hyptis suaveolens Poit. (Lamiaceae)

Imperata cylindrica L. (Poaceae)

Ipomoea eriocarpa L. (Convolvulaceae)

Leucas martinicensis (Jacquin) R. Brown

(Lamiaceae)

Paspalum scrobiculatum Berg (Poaceae)

Pennisetum pedicellatum Trin. (Poaceae)

Pennisetum polystachion L. (Poaceae)

Phyllanthus amarus Sch. et Th. (Euphorbiaceae)

Rottboellia cochinchinensis Loureito (Poaceae)

Sida acuta Brum. F. (Malvaceae)

Sida urens Brum. F.(Malvaceae)

Spermacoce chaetocephala De Candolle (Rubiaceae)

Spermacoce radiata De Candolle (Rubiaceae)

Spigelia anthelmia (Loganiaceae)

Stylochiton hypogaeus Lepr. (Araceae)

Tephrosia bracteolata Guil. Perr. (Fabacaceae)

Tricliceras pilosum (Will) Persoon (Turenaceae)

Tridax procumbens L. (Asteraceae)

Waltheria indica L. (Sterculiaceae)

Zornia glochidiata Reichb. ex DC (Fabacaceae) 